\title{
Theoretical-Probability Model of Metal Removal During Magnetic-Abrasive Treatment
}

\author{
Alexey Ikonnikov and Sergey Leonov* \\ I.I. Polzunov Altai State Technical University, 656038 Barnaul, 46 Lenin Avenue, Russian Federation
}

\begin{abstract}
The work is devoted to the issue of calculating material removal during magnetic abrasive processing. Cutting grains have random dimensional characteristics, are randomly located on the surface of the tool, the workpiece has an irregular profile. The cutting parts of the grain tops partially remove the chips, and partially elastically-plastic deform the metal. Part of the vertices falls into the risks on the surface of the workpiece formed by the previous machining, and part - into the risks from passing through the previous vertices. This process is determined by the probability of the contact of the top of the grain with the metal. The developed stochastic models make it possible to predict the removal of metal from the treated surface depending on the time and parameters of the operation, which creates the prerequisites for their use in the design of polishing operations.
\end{abstract}

\section{Introduction}

Any method of abrasive processing is characterized by the stochastic nature of metal removal. Cutting grains have random dimensional characteristics, are randomly located on the surface of the tool, the workpiece has an irregular profile. Here are just some of the factors that cause the appearance of random components in the profile of the treated surface. Therefore, the parameters of the surface roughness of the part, and the removal of metal during magnetic abrasive processing are also stochastic parameters.

A probabilistic theoretical model of metal removal and roughness formation during magnetic abrasive processing includes two subsystems: "cutting process" and "output function". The last subsystem forms the output indicators of the process (Fig. 1).

The result of the functioning of the subsystem "cutting process" is the profile of the processed surface. The formation of the profile is determined by the interaction of the magnetic abrasive powder, compacted by a magnetic field, with the treated surface. In this case, the cutting forces providing this interaction are the input parameters of the subsystem. Modeling the process of removing material from the surface of the workpiece must begin with a description of the phenomena of microcuts by individual grains [5].

Abrasive grains have a complex composition and microrelief. The shape of the grain depends on the method of their manufacture. The grains vary in shape, have a different number of cutting peaks, and their rounding radii differ. Therefore, there is a random

\footnotetext{
*Corresponding author: sergey_and_nady@mail.ru
} 
distribution of cutting vertices over the surface and depth of the grain conglomerate. In addition, the radii of curvature at the cutting vertices are also randomly distributed. The depth of penetration of grains into the workpiece material depends on these radii, as well as on the cutting force and microhardness of the treated surface.

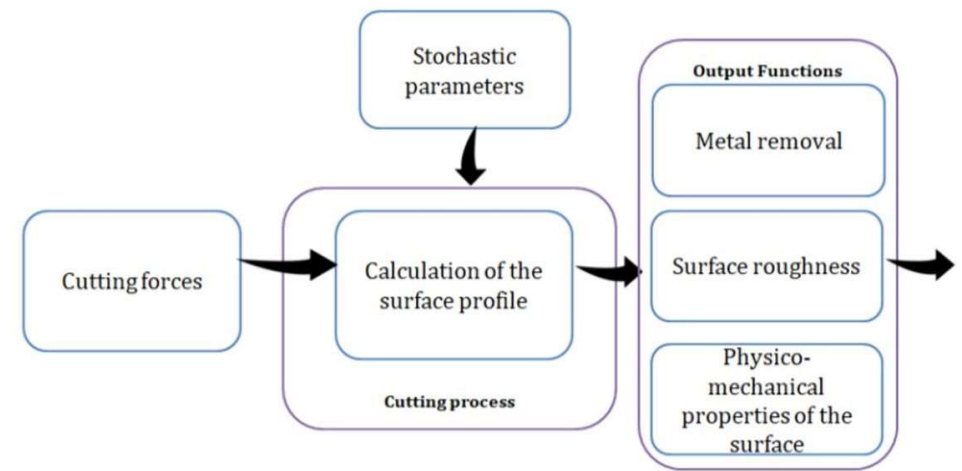

Fig. 1. The structure of the probability theory model

The cutting parts of the grain tops partially remove the chips, and partially elasticallyplastic deform the metal. The resulting risks are superimposed on an already created profile. Therefore, when the grain passes through the zone of contact of the tool with the workpiece, the cutting part of the grain contacts the metal only part of the time. Part of the vertices falls into the risks on the surface of the workpiece formed by the previous processing, and part - into the risks from passing through the previous vertices [15]. This process is determined by the probability of the contact of the top of the grain with the metal.

\section{Research methodology}

The random value is most fully determined by its distribution function or its derivative distribution density. If the laws of distribution of random variables at the input of the system are known, they can be recalculated into the laws of distribution at the output, and, therefore, the average values and the spread of the output parameters can be determined [4, 14].

There are 2 approaches for this recount:

1. The analytical approach. If the analytical expressions for the distribution functions of the input parameters are known, and the analytical dependencies that form the output parameters from the input parameters are known, then one can determine the analytical dependencies that describe the distribution of these output parameters. It is not always possible to obtain analytically the law of distribution. But if there are many input random factors, then the output parameters are distributed close to the normal law. In this case, it is sufficient to determine only the distribution parameters: average value and variance.

2. Monte Carlo method (stochastic modeling). It is not always possible to even approximately recount the laws of distribution of input parameters into the laws of distribution of output. In this case, sets of random values of the input parameters are generated, the values of the output parameters are calculated for them, and then the form and parameters of this law are restored from the histogram or polygon of the distribution density.

The Monte Carlo method is simple. With its software implementation, cycles of generating random input parameters and a module for constructing histograms and their analysis are added to the mathematical model. The only drawback of this approach is its 
complexity. To obtain histograms (polygons) and reliably restore the form of the distribution law from them, it is necessary to perform at least several hundred "runs" using a mathematical model. If the time of a single simulation is, for example, $1 \mathrm{~s}$, then when using the Monte Carlo method, the total calculations will require about $1000 \mathrm{~s}$ - more than 15 minutes. The increase in productivity of modern computer technology and the use of parallel computing methods in multicore and multiprocessor systems somewhat mitigate this drawback.

The analytical approach is actually the opposite of the Monte Carlo method. Its main advantage is high speed: the distribution law and its parameters are obtained on the basis of analytical dependencies without any "runs". However, obtaining these dependencies may require considerable labor at the preliminary stage. Moreover, it is not always possible to obtain these dependencies using exact methods and one has to make simplifications (for example, approximating complex analytical dependencies), which may somewhat reduce the accuracy of calculations. But the high speed when using analytical stochastic models makes this approach more preferable.

\section{Results and discussion}

Magnetic abrasive treatment is mainly designed to remove the initial roughness of the workpiece (polishing) [2, 8, 12, 13]. Therefore, it is first necessary to consider its initial profile and the process of its transformation into a profile formed during polishing.

The initial surface profile for finishing and finishing operations is formed during finishing milling. For milling complex surfaces, spherical milling cutters are most often used and three typical sections can be distinguished (Fig. 2). The profile of microroughness depends on the radius of the cutter and step S [11].

$\mathrm{n}$

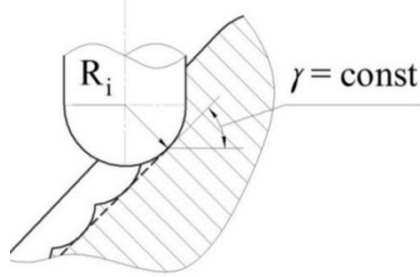

a)

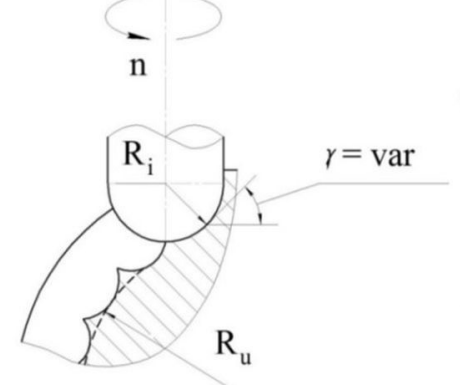

b)
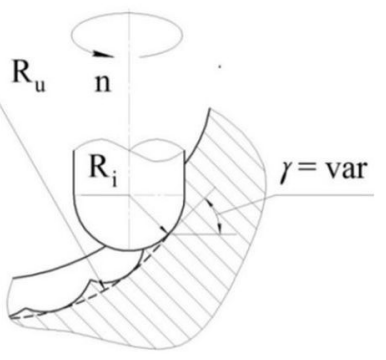

c)

Fig. 2. Typical sections of milling complex surfaces: a) a flat area; b) a convex section; c) concave section

Given the possibility of plastic deformation of the metal during milling, mathematically, the shape of the risks can be described by a power law dependence

Maximum height risks

$$
\mathrm{u}=\mathrm{ax}^{\mathrm{b}} \text {. }
$$

$$
u_{\max }=a(S / 2)^{b} \text {. }
$$

To describe the surface profile during abrasive treatment, Yu.K. Novoselov [1, 7] suggested using the probability of material removal. Depending on the level of $y$ and processing time $\tau$, this probability is calculated by the formula: 


$$
\mathrm{P}_{\mathrm{M}}(\mathrm{y}, \tau)=1-\exp \left(-\mathrm{a}_{0}(\mathrm{y}, \tau)-\mathrm{a}_{1}(\mathrm{y})\right),
$$

where $a_{0}=k_{c} \lambda b_{3}-$ characterizes the formation of the profile in the contact zone of the tool with the workpiece;

$a_{1}$ - characterizes the surface profile of the workpiece before processing;

$\mathrm{k}_{\mathrm{c}}-$ is the coefficient of chip formation;

$\lambda$ - the number of vertex grains that have passed a given section of the workpiece;

$b_{3}-$ is the average value of the grain width at the level of the conditional outer surface of the workpiece;

$\tau$ - time.

For the workpiece, $\mathrm{a}_{0}=0$ and the probability of material removal, which describes its profile, is determined by the dependence $P_{M}(y)=1-\exp \left(-a_{1}(y)\right)$. Fig. 3 shows the dependence $\mathrm{P}(\mathrm{y})$.

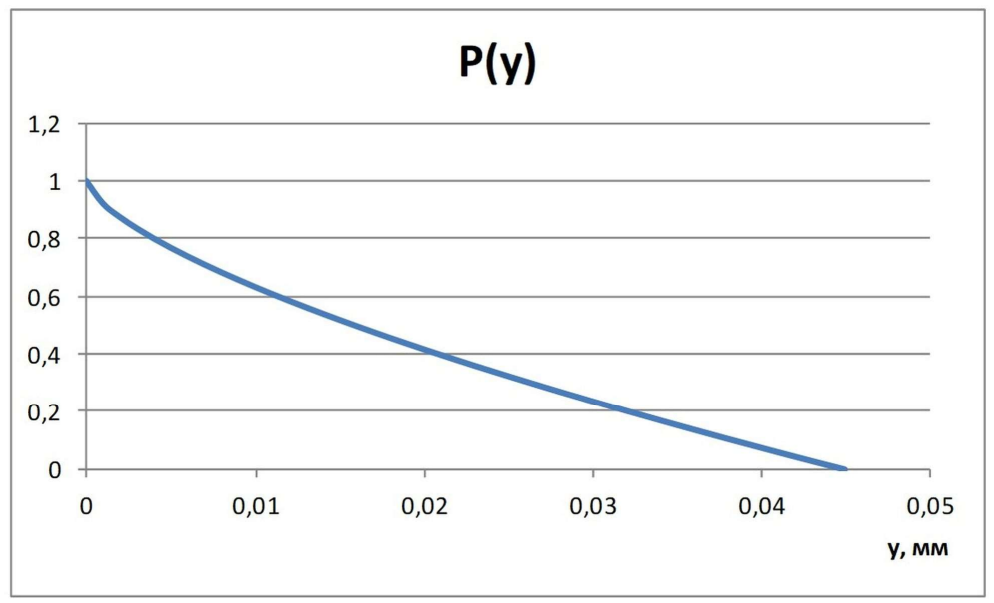

Fig.3. The probability of metal removal for the initial risk after milling

In magnetically abrasive processing, metal removal occurs mainly from the top of the risks and the roughness introduced by the process is initially substantially less than the height of the risks after milling. In fact, we can assume that the top of the risks is cut off and the resulting profile has the form shown in Figure 4.

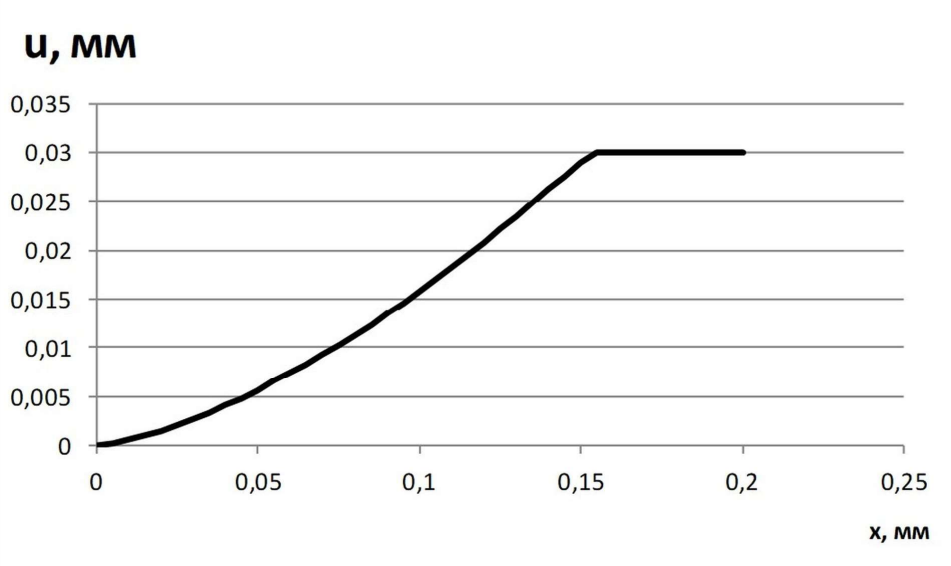


Fig. 4. Form of risks after removal of part of the metal

In this case, the roughness of the horizontal platform is initially substantially less than the roughness of the entire surface. Moreover, the probability of metal removal for small values of y decreases with a simultaneous decrease in height risks [9, 10]. To determine the amount of metal removal $\Delta$ from the surface of the workpiece, we consider the process of interaction of the tool and the workpiece at discrete points in time $\tau_{\mathrm{K}}$, corresponding to the output of the magnetic circuit with grains from contact with the workpiece - Figure 5 [6]. In this case, the contact probability value (3) is determined by the coefficient $a_{0}\left(y, \tau_{\kappa}\right)$. Assuming that the vertices of the grains are distributed in accordance with the density $f(u)=\frac{1,5}{t_{\phi}^{1,5}} \sqrt{u}$, and the profile of the top of the grain is described by the dependence $b_{3}=$ $2 \sqrt{2 \rho\left(t_{\phi}-y-u\right)}$, expression for this coefficient at the moment the magnetic circuit comes out of contact with the workpiece $\tau_{\mathrm{K}}$ (Fig. 5) takes the form:

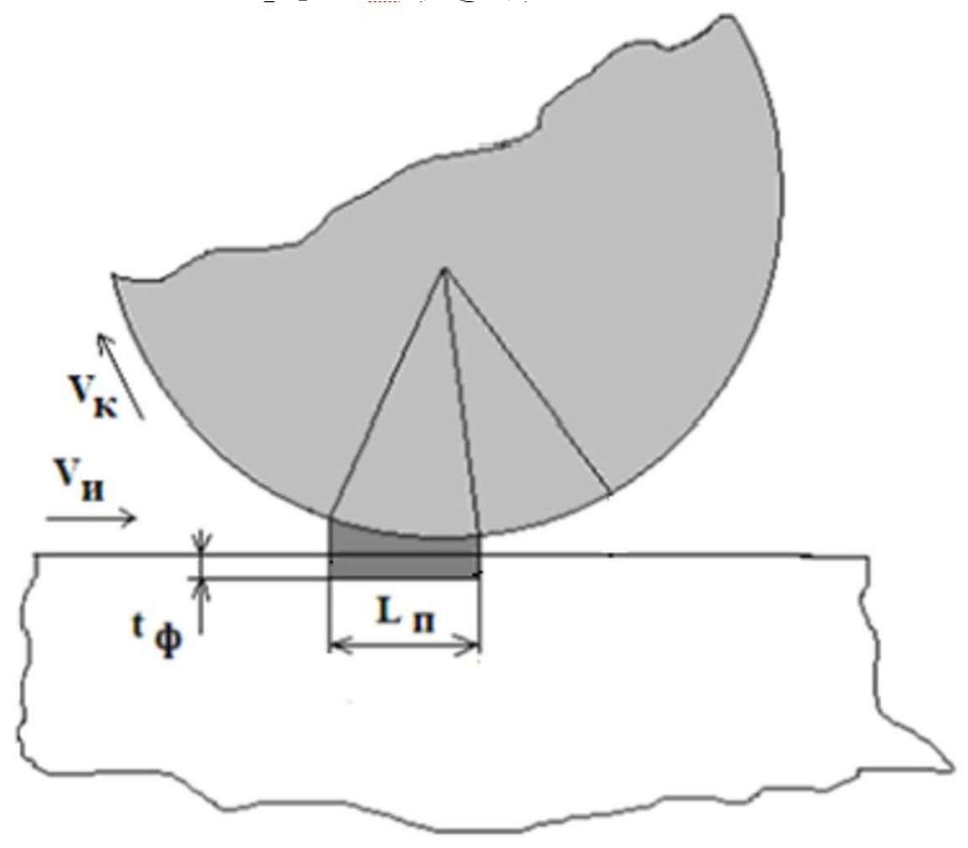

Fig. 5. Scheme of contact of the tool with the workpiece

$$
a_{0}\left(y, \tau_{\kappa}\right)=\frac{3 \pi k_{c} n_{3}}{8 t_{\phi}^{1,5}}\left(t_{\phi}-y\right)^{2} L_{\Pi} \sqrt{2 \rho}
$$

where $t_{\phi}$ - is the maximum depth of cut, $n_{3}-$ is the number of grains per unit surface of the tool, $\rho-$ is the average radius at the top of the grain, $k_{c}-$ is the chip formation coefficient.

When passing through the workpiece section of several magnetic cores with grains, formula (3) takes the form:

$$
P_{M}\left(y, \tau_{k}\right)=1-\exp \left(-a_{0}\left(y, \tau_{k}\right)-a_{1}\left(y_{1}\right)-a_{2}\left(y_{2}\right)-\ldots-a_{n}\left(y_{n}\right)\right),
$$

where $a_{0}$ - characterizes the formation of the profile in the contact zone of the tool with the workpiece in the current passage; $a_{1}, a_{2}, \ldots$ - characterize the surface profile of the workpiece in previous passes; $\mathrm{n}-$ is the number of passes.

For the outer surface of the workpiece, $\mathrm{y}=\Delta, \mathrm{y}_{\mathrm{k}}=(\mathrm{k}+1) \Delta$. Moreover, the probability $\mathrm{P}_{\mathrm{M}}$ $=1-\beta$. Expressing the equation obtained from (5) in dimensionless form, we obtain: 


$$
\sum_{k=1}^{[1 / X]}(1-k X)^{2}=F
$$

where $\mathrm{X}=\Delta / \mathrm{t}_{\phi}$. When $\beta=0,0027$ we obtain: $F \approx \frac{3,550}{k_{c} L_{\Pi} n_{3} \sqrt{\rho t_{\phi}}}$

Equation (6) is solved with respect to $X$ by a numerical method. We have obtained approximate analytical expressions for solving this equation. Experimental verification confirms the adequacy of the dependence and the correctness of the approach used.

\section{Conclusions}

1. The analytical approach to constructing stochastic models can significantly reduce the time for predicting the quality indicators of magnetic abrasive processing, providing the necessary accuracy of calculations.

2. Magnetic abrasive treatment is most often used to polish milled surfaces across the edges. In this case, the removal of metal mainly occurs from the vertices of the scribes until their height is comparable with the roughness introduced by the polishing itself.

3. The developed stochastic models make it possible to predict the removal of metal from the treated surface depending on the time and parameters of the operation, which creates the prerequisites for their use in the design of polishing operations.

\section{References}

1. Y. Novoselov, V. Bogutsky, L. Shron, A. Kharchenko, MATEC Web Of Conferences Cep. "International Conference on Modern Trends in Manufacturing Technologies and Equipment, ICMTMTE 2017", 01080 (2017)

2. Biing-Hwa Yana, Geeng-Wei Changa, Tsung-Jen Chenga, Rong-Tzong Hsub, Journal of Machine Tools \& Manufacture, 43, 1355 (2003).

3. Dhirendra K. Singh, V.K. Jain, V. Raghurama, R. Komanduri, Wear 259, 1254 (2005) 1254-1261.

4. Dhirendra K. Singh, V.K. Jain, V. Raghuram, Journal of Materials Processing Technology, 149. 22 (2004)

5. B. Girma B, S.S. Joshi, M.V. Raghuram, R. Balasubramaniam, Machine Sci Technology, 10 (3), 32334 (2006).

6. Harish Kumar, Sehijpal Singh, Pardeep Kumar, Journal Of Engineering Research \& Technology (IJERT), 2, 3, (2013)

7. Y. Novoselov, V. Bogutsky., L. Shron, Procedia Engineering Cep. "International Conference on Industrial Engineering, ICIE 2017", 991 (2017)

8. S.C. Jayswal, V. K. Jain, P.M. Dixit, International Journal of Advanced Manufacturing Technology, 26, 477 (2005)

9. Junmo Kanga, Andrew Georgea, Hitomi Yamaguchia, Procedia CIRP 1414 (2012 )

10. K. Singh, Dhirendra \& Jain, Vijay \& Raghuram, V.. Journal of Materials Processing Technology, 149, 22, (2004)

11. Krishankant, Jatin Taneja, Mohit Bector, Rajesh Kumar, International Journal of Engineering and Advanced Technology (IJEAT), 2, 1, (2012)

12. Baljinder Singh, International Research Journal of Engineering and Technology (IRJET), 2, 04 (2015)

13. Hitomi Yamaguchia, Anil K. Srivastavab, Michael A. Tana, Journal of Manufacturing Technology, 61, 311 (2012) 
14. T.C.Kanish, P.Kuppan, S.Narayanan, S. Denis Ashok, Procedia Engineering, 97, 1948 (2014).

15.E.Y. Tatarkin, A.M. Ikonnikov, T.A. Schreiner, R.V. Grebenkov, Applied Mechanics and Materials, 788, 69 (2015) 